\title{
Imaging Techniques for Cancer Diagnosis and Scope for Enhancement
}

\author{
Showkat Hassan Malik ${ }^{1}$ \\ ${ }^{1}$ Department of computer Science, Mewar University, Rajasthan, India \\ E-mail: shmalik.mca@gmail.com \\ Tariq Ahmad Lone ${ }^{2}$ \\ ${ }^{2}$ Department of computer Science, Mewar University, Rajasthan, India \\ E-mail: tariq380@gmail.com \\ Prof. S. M. k Quadri ${ }^{3}$ \\ ${ }^{3}$ Department of computer Science, Jamia Millia Islamia University, New Delhi, India \\ E-mail: Quadrismk@hotmail.com
}

\begin{abstract}
Imaging techniques are used to create images of structure, function and pathology of human body organs for cancer diagnosis. Various imaging techniques like X-ray, Positron Emission Tomography (PET), Ultrasound (US), MRI etc. are used for cancer diagnosis. These imaging techniques have gone through Lot of advancements during last few years. These techniques vary in the technology and application. Various artifacts exist in these imaging techniques and images produced by theses imaging techniques. These artifacts can be exploited to enhance the imaging technique and the images produced by them.
\end{abstract}

Index Terms-Imaging techniques, cancer diagnosis, image enhancement, image artifacts.

\section{INTRODUCTION}

Imaging techniques create visual representation in the form of images of interior of body to reveal the structure, function and pathology of human body organs for cancer diagnosis and treatment. There are several imaging techniques to view the internal structure of human body, each type gives different information about the organ of the human body being treated or studied for effective medical diagnosis and treatment. Hidden structure of bones and skin is revealed using Imaging Techniques. Imaging Techniques are not only used for detection but also for determining the stage and location of cancer in order to provide aid in treatment and surgery. There are various uses of imaging as for as cancer is concerned like screening of cancer: imaging is used to check abnormalities that might be cancerous. Diagnosis: imaging can be used to find out the location of cancer and how much it has spread. Guiding cancer Treatment: imaging can be used to focus on the exact location of cancer during treatment and determine whether treatment is working or not. Many imaging techniques are used for cancer diagnosis, treatment and planning surgery like $\mathrm{X}$ - ray, Ultrasound (US), Positron Emission Tomography (PET), Single-Photon Emission Computed Tomography (SPECT), Computed Tomography (CT), Magnetic Resonance Imaging (MRI), Optical Imaging (OI) etc. Medical X-rays are very important to help diagnose different diseases, including cancer. X-rays and CT scans use ionizing radiation which can lead to health risks from the radiation but the use of these equipments are low and the benefits overweight the risk as far as right diagnosis and treatment is concerned. Ultrasound imaging technique can help a physician to evaluate, diagnose and treat medical conditions like to visualize abdominal tissues and organs, assess bone fragility, visualize breast tissue, listen to the fetal heart beat, visualize blood flow through a blood vessel, organs, view the heart, view the fetus in pregnancy, visualize ocular structures etc. PET and SPECT imaging techniques allows for the study of molecular and cellular events in the living organism. PET and SPECT can be made well suited for molecular imaging of cancer. MRI imaging technique is also important that can help physicians in analyzing various diseases including tumors. MRI has high accuracy as compared to CT scan. MRI can be used for analysis of local diseases and can provide valuable information about breast conditions that may not be obtained by other imaging techniques, such as mammography or ultrasound. Optical imaging is cheap and fast imaging technique. OI is used to detect human cancers in locations that are accessible by an optical imaging device. Molecular function of cancer cells of human body can be investigated using Light. Advancements in instrumentation of OI make it possible to investigate tissue of several centimeters in depth. The use of optical imaging is now being applied in operating room for the localization of malignant tumors in addition to screening of cancer. These techniques are discussed in detail in the following sections [III-X]. There exist various artifacts in these imaging techniques. Artifact is misrepresentation of tissues in the output image. The artifacts produced by them result in difficulties in diagnosis by radiologists. 
This paper is organized as: Section II presents related work, III-X presents the different Imaging techniques and enhancement issues and Section XI Presents conclusion of the paper.

\section{RELATED WORK}

In this section we briefly present imaging techniques for cancer diagnosis. Imaging technique is one of the most important tool for evaluating size and form of internal body structures. The aim of imaging techniques is to aid in diagnosis and treatment of various diseases especially cancers and tumors. There are various imaging techniques which can be used for cancer diagnosis and guiding treatment like X-rays, US, PET, SPECT, CT, MRI, OI etc. $\mathrm{X}$-ray typically was the first imaging technique used to help diagnose symptoms of various diseases like shortness of breath, persistent cough, injury, lung cancer etc. Since in the middle of the 1990s, X-ray imaging has been increasing attention. The advantage of X-ray is that high sensitivity is achieved for weak absorbing material, such as soft tissues, which generate a poor contrast by other imaging techniques. Medical and biological imaging is the main target of X-ray imaging. The progress of X-ray imaging depends on advances in third-generation synchrotron radiation source, optical element, and image detector [47] [53]. US have undergone dramatic changes since its invention three decades ago. US have evolved into high resolution real-time imaging system. Advances in Electronics and computing have revolutionized ultrasound imaging applications. Advances in Digital signal processing has innovated beam forming, image display and archiving [48] [54-55]. SPECT developed in 1960 was used for clinical practice in 1980. SPECT and its competitor PET is used for acquisition of nuclear medicine image data. Technical advances in electronics and reconstruction algorithms have evolved SPECT and PET considerably [49] [56]. MRI whole body scanner developed in 1980 is used to get an image of the anatomy and the physiology of human body. Advances in MRI image analysis have increased the utility of MR in diagnosis, screening, management, and therapy monitoring of cancer [50] [57]. OI allows threedimensional reconstruction of optical signals in human body using diffuse optical tomography. These technological advances in imaging helps in investigating the molecular functions of tumors [51] [58].

\section{X-RAY}

An X-ray developed in 1895 represents major advances in medical science. X-ray imaging examinations are recognized as a valuable medical tool for a wide variety of procedures. They are used to support medical and surgical treatment planning. X-rays use electromagnetic radiation to make images on a film called radiograph. X-rays are passed through human body to show images of bones, tumors and dense matter. Some rays are absorbed and some are attenuated. Dense tissues can cause more attenuation resulting in white color in x-ray image while as less dense tissues can cause less attenuation resulting in darker spots in X-ray image [3-5]. X-ray can be used for cancer diagnosis and staging and guiding treatment. $\mathrm{X}$ rays can also be used to destroy cancer cells using radiation therapy. There are different types of x-rays like chest $\mathrm{X}$-ray; bone $\mathrm{X}$-ray etc. X-rays help doctors to diagnose cancer, to get size of tumor, to compare tumors before and after treatment. Some x-ray images are shown in figure 1 below.

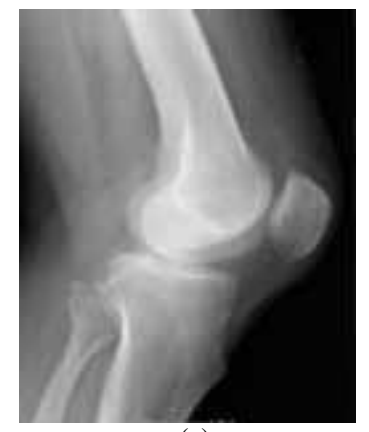

(a)

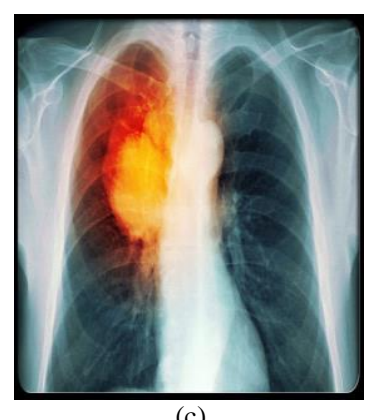

(c)

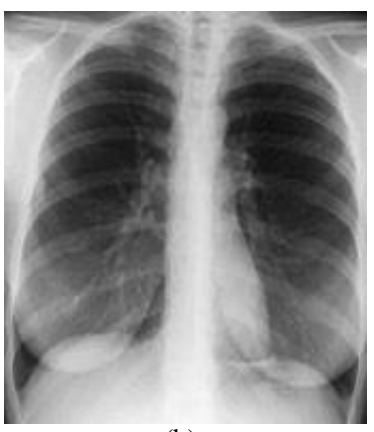

(b)

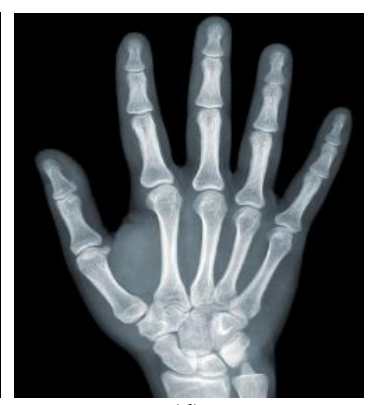

(d)
Fig.1. (a) X-ray of Knee, (b) Chest X-ray, Lung Cancer X-ray, (d) X-ray of Hand

\section{A. X-ray Artifacts}

Various Artifacts may occur in X-ray images due to improper handling of the film packet. The movements also result in various artifacts due to excessive movement of the tube, the movement of patient or the film. X-rays images may be distorted due to improper alignment of the tube, object or film, finger prints, blurred images, cone cutting, double exposure etc.[6].

$\mathrm{X}$-ray as an imaging technique has some advantages and disadvantages:

\section{ADVANTAGES}

1. X-ray procedure is quick and painless.

2. X-ray helps in disease diagnosis, including some cancers, infections and broken bones.

\section{DISADVANTAGES}

1. Children have greater risk of cancer due to exposure to ionizing radiation (x-rays).

2. Soft tissue tumors do not show well on $\mathrm{x}$-ray image.

3. X-ray images cannot always differentiate between cancerous and non cancerous tumors. 


\section{MAMMOGRAPHY}

Among the female population breast cancer is the second most frequent malignant neoplasm. Mammography is used to detect breast disorders. The goal of mammography is early detection of breast cancer. In mammography special X-ray scan technique in which low energy X-rays are used to examine human breasts in order to detect masses, micro calcifications and breast cancer. The process of obtaining mammograms is almost same as that of x-ray image, the difference being that only low energy $\mathrm{x}$-rays are used in mammograms as compared to normal x-rays. In mammography in order to get an image the breast is compressed using a dedicated mammography unit to make the thickness of breast tissue even. Even thickness of breast tissue increases the image quality because low energy $\mathrm{x}$-rays pass through easily. The breast is held still and images of the breast are taken. There are various types of mammography like film mammography, digital mammography etc. digital mammography has higher cancer detection rate as compared to film mammography. The characteristics of the tumors are same using both the methods [2] [7] [9]. Main aim of mammography is to detect breast cancer and guide treatment. Besides mammography there are other techniques used for detection of breast disorders like US, MRI and positron emission mammography (PEM). Some mammogram images are shown in figure 2 below.

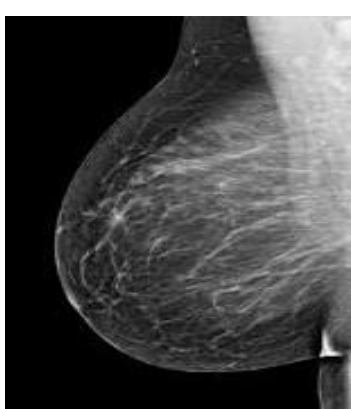

(a)

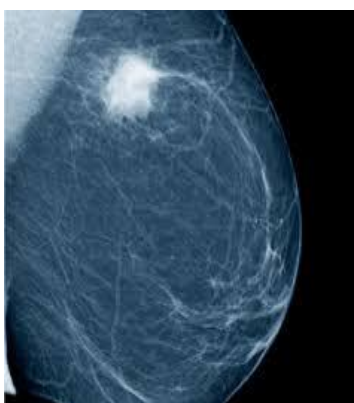

(c)

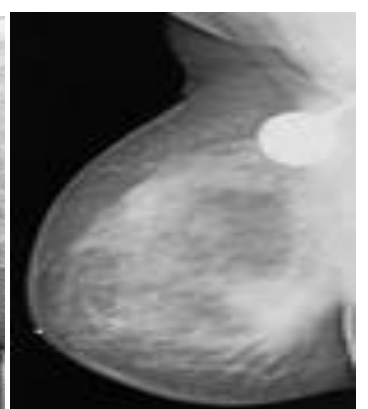

(b)

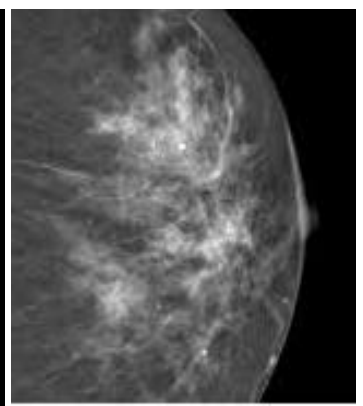

(d)
Fig.2. (a) (b) Mammograms, (c) (d) Breast Cancer

\section{A. Mammography Artifacts}

Artifacts in mammograms images reduce image quality and results in technical difficulties for the radiologist to interpret the results. The artifacts in mammography can be categorized as Detector like artifacts, machine related artifacts, patient related artifacts, storage related artifacts, and processing related artifacts. These artifacts can be reduced by stimulating them by using some enhancement techniques for better results and diagnosis. These artifacts can be recognized to improve the quality of mammographic interpretation and to prevent the characterization of artifacts as breast disease [8-11].

Mammography has some advantages and disadvantages when used for diagnosis breast related diseases:

\section{ADVANTAGES}

1. Mammography procedure is quick and painless.

2. It reduces mortality by early detection of breast cancer.

3. It helps in improving treatment and can enhance the life span of patients [12].

\section{DISADVANTAGES}

1. Exposure to ionizing radiation

2. False detection of breast cancer which leads to further tests.

3. All cancers cannot be detected

4. Can lead to over diagnosis.

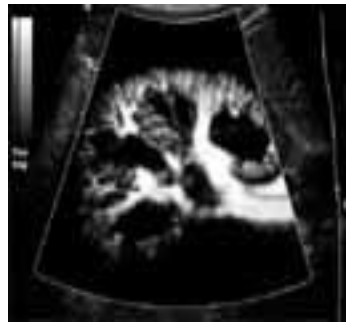

(a)

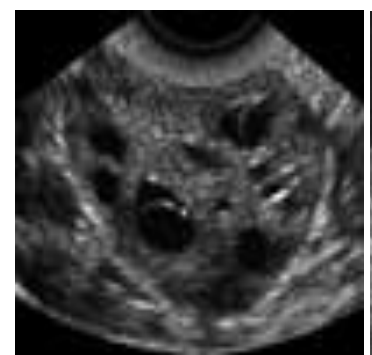

(c)

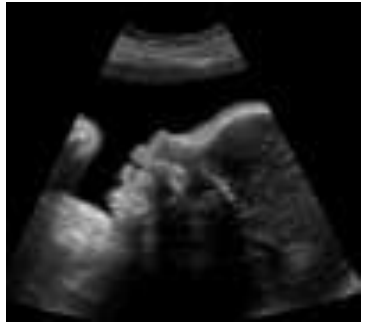

(b)

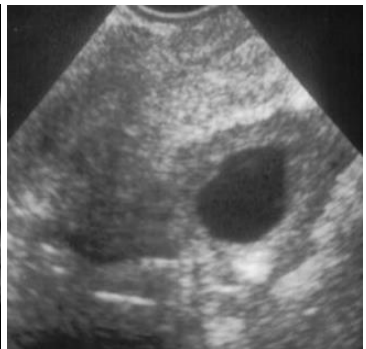

(d)
Fig.3. (a) US of Kidney, (b) US of Unborn Baby, (c) Us of Ovary, (d) US of Ovarian Tumor

\section{ULTRASOUND (US)}

In US high frequency sound waves are used to produce images of interior organs and tissues of the body. In order to perform ultrasound examination the patient needs to apply a thin layer of gel to the skin. A transducer is directly placed on the skin to transmit the waves through the gel into the body. The reflection of sound waves from the body parts, the strength of the signal and the transmission time is used to produce the images. Ultrasonic imaging uses $2 \mathrm{MHz}$ and higher frequencies to locate a tumor inside the body. During an ultrasound a handheld device is placed on the body and moved around 
then images of internal organs can be seen on the monitor. US advances include three dimensional US and Doppler US. Three dimensional US converts sound waves into three dimensional images. Doppler US can be used to show direction and speed of blood flowing through veins [14]. Ultrasound images help doctors to diagnose and treat medical conditions. Ultrasound also helps to perform biopsy by showing tumor location in the body [15]. Some Ultrasound images are shown in figure 3 below.

\section{A. Ultrasound artifacts}

Ultrasound (US) Images encounter various artifacts which can be the source of confusion for interpreting results by the radiologists. Some artifacts may be avoidable and arise due to improper scanning technique. Other artifacts are generated by the physical limitations of the modality. US artifacts can be understood with a basic appreciation of the physical properties of the ultrasound beam, the propagation of sound in matter, and the assumptions of image processing. US artifacts arise due to errors inherent to the ultrasound beam characteristics, the presence of multiple echo paths, velocity errors, and attenuation errors. Artifacts are encountered routinely in clinical practice. Recognition of these artifacts is important because they may be clues to tissue composition and aid in diagnosis [16]. The ability to recognize and remedy potentially correctable US artifacts is important for image enhancement and optimal patient care. Artifacts in ultrasonic images can be classified into three categories:

1. Artifacts related to instrument problems, which occur when the equipment is not functioning satisfactorily.

2. Technique dependent artifacts, in which artifacts are produced by unsatisfactory Operator technique.

3. Artifacts due to the way tissues affect sound [17].Ultrasound imaging technique has some advantages and disadvantages:

\section{ADVANTAGES}

1. Ultrasound is safe because no ionizing radiations are used.

2. Soft tissues and organs can be visualized.

3. US can detect lesions in dense breasts when mammograms cannot.

4. US can differentiate between cyst and solid mass.

5. US can be used to detect flow of blood through veins.

6. US can be used to diagnose medical condition of pregnant women when other imaging techniques are not safe.

7. US can be used to check health of unborn baby.

\section{DISADVANTAGES}

1. US require a highly experienced expert to perform ultrasound examination.

2. Some cancers cannot be detected by ultrasound.

3. Patients may require preparation for the ultrasound examination.

\section{POSITRON EMISSION TOMOGRAPHY (PET)}

PET creates images of body and organs. The patient is injected radioactive substance linked to components used by body cells or components that are absorbed by cancer cells. Patient lies still for an hour while radioactive substance circulates through body. If patient is having tumor the radioactive substance gets accumulated in the tumor. The PET scanner captures the distribution of radioactive substance in the body [18-19]. The computer then translates the distribution of radioactive substance into an image which then is interpreted by a radiologist, to detect tumor given the fact that cancer cells absorb more radioactive substance than other tissues in the body. PET scan helps in determining whether a mass is cancerous or not. PET scan shows accurate results for large tumors and can detect cancer while other imaging techniques may show normal results. PET scan also helps in checking whether treatment is working or not. Some PET images are shown in figure 4 below.

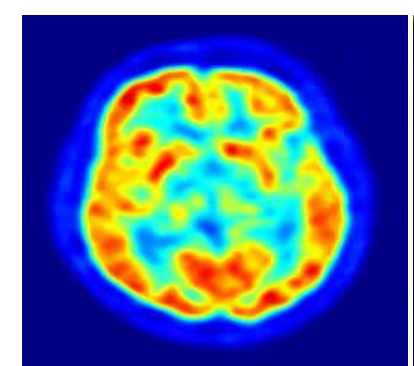

(a)

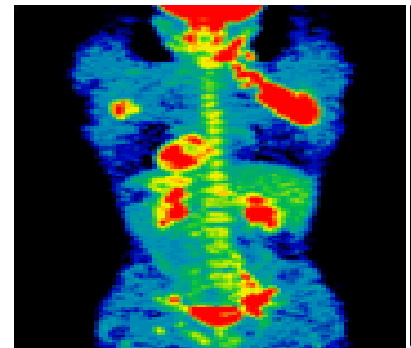

(a)

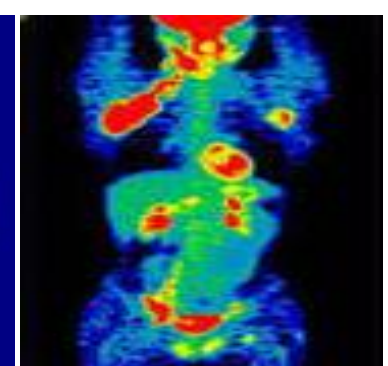

(b)

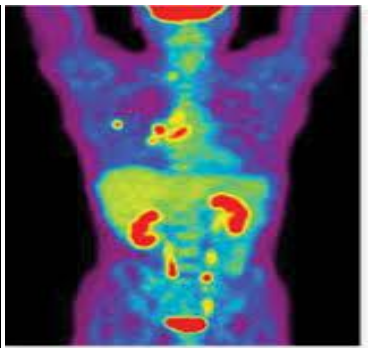

(b)
Fig.4. (a) PET scan of Human Brain, (b) (c) (d) PET Scan of Human Body

\section{A. PET Artifacts}

A Positron Emission Tomography (PET) scan is performed to detect the function and activity of the cells in human body. It can be used to evaluate diseases in which there is a change in the cells such as cancer. PET image artifacts are caused due to: metallic implants, respiratory motion, contrast medium, and truncation. There can be errors due to Calibration problems, Detector failures, Resolution and partial volume effects and patient motion [20-26]. Various advantages and disadvantages associated with PET scan technique are:

\section{ADVANTAGES}

1. PET scan enables us to detect disease before apparent changes in the anatomy take place.

2. PET differentiates between cancerous and non cancerous tumors. 
3. PET can be used to plan surgery and to check the overall spread of the diseases.

4. PET is very effective in diagnosing early stages of cancer and neurological diseases.

\section{DISADVANTAGES}

1. PET scan is expensive, therefore is not offered in all the medical institutions in the world.

2. PET scan is risky for some patients like pregnant women because of radioactive substance used in the procedure.

3. Patients can go through PET scan procedure limited number of times due to use of radioactive substance.

\section{SINGLE-PHOTON EMISSION COMPUTED TOMOGRAPHY (SPECT)}

SPECT also uses radioactive substance to be injected in the body through veins. The SPECT scanner is used to capture the flow of blood and chemical reaction in the body to make computerized 2D, and 3D images. Radioactive substance gets stick to tumor cells which may be detected by SPECT scanner to diagnose cancer. SPECT is a dynamic field which has improved a lot during last few years both in hardware technology and image processing algorithms. The components used in SPECT like Scintollators and photon transducers have also improved. The new advance in SPECT includes highcount sensitivity cardiac SPECT systems and hybrid SPECT/CT systems. The reconstruction algorithms have also gone through advancements in order to minimize image acquisition time and at the same time keeping the diagnostic quality. In nuclear medicine Preclinical smallanimal SPECT systems have become a major focus to follow treatment [27-28]. Some SPECT images are shown in figure 5 below.

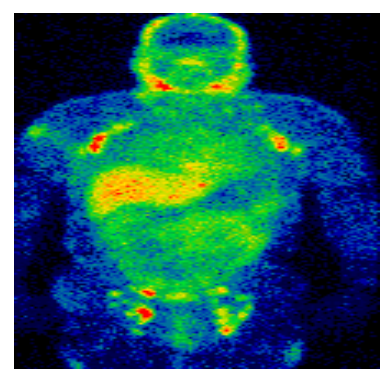

(a)

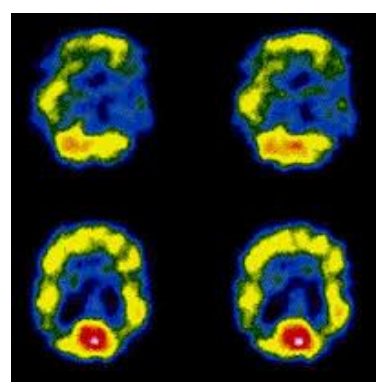

(c)

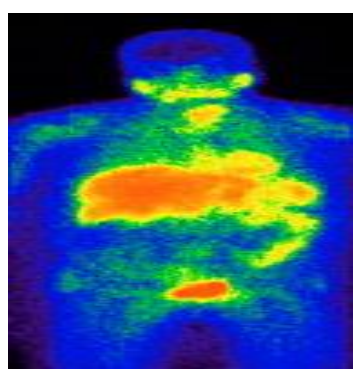

(b)

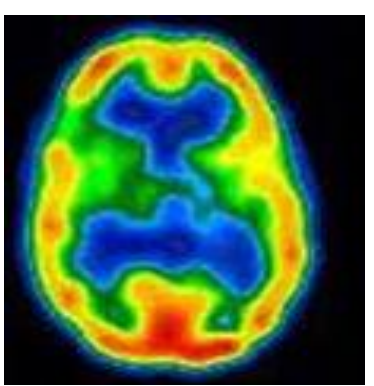

(d)
Fig.5. (a) (b) SPET Scan of Human Body, (c) (d) SPECT Scan of Brain

\section{A. SPECT Artifacts}

In SPECT there are certain artifacts that may cause attenuation in the image when there is a case of cancer diagnosis. The artifacts that may arise are somehow similar to PET artifacts like Motion artifacts, Scatter, low contrast, metal induction, low radiation dose for malignancy detection etc. These artifacts need to reduce these for better image quality for early diagnosis [29-30].

SPECT advantages and disadvantages are as under:

\section{ADVANTAGES}

1. SPECT scan can detect disease before apparent changes in the anatomy take place.

\section{DISADVANTAGES}

1. SPECT scan is very time consuming procedure

2. Images produced by SPECT are low in resolution.

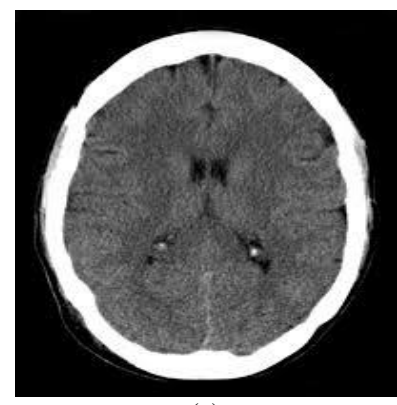

(a)

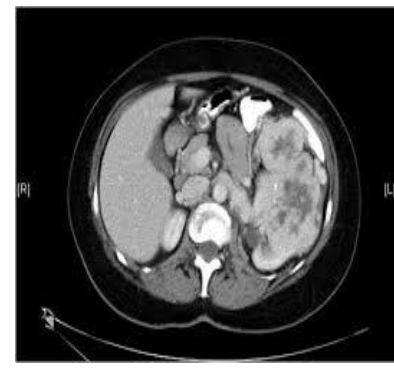

(c)

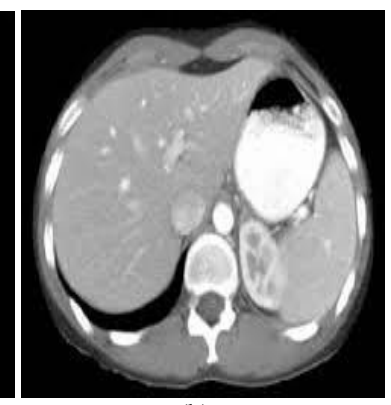

(b)

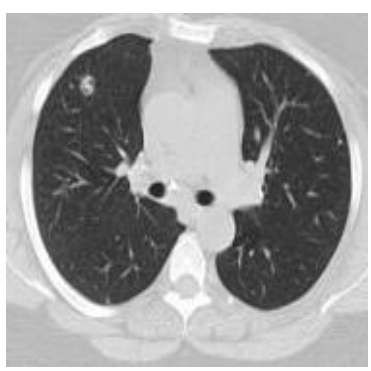

(d)
Fig.6. (a) CT Image of Brain, (b) CT Image of Liver, (c) CT Image of Kidney, (d) CT Image of Lung

\section{COMPUTED TOMOGRAPHY (CT)}

CT uses $\mathrm{x}$-ray equipment to create cross-sectional layers that show detailed images of the internal body organs, including bones, tissues, and tumors. During CT procedure series of images are created like a loaf of sliced bread which can be looked at individually. These 2D sliced images can be combined to create 3D images. CT Images help doctors to diagnose various diseases especially cancer. CT images can show information which experienced radiologists can use to get approximate location of tumor CT helps doctors to detect abnormal tissue growth, diagnose the presence and recurrence of a tumor, provide information about the stage of a cancer, guide treatments and find whether a cancer is responding to treatment, to help plan radiation therapy or surgery. 
Conventional CT scan takes series of images of slices of body. These slices are few millimeters apart. Recent technical advances in CT scan increase the effectiveness and speed by taking continuous pictures of body so that there is no gape in the pictures collected [31-35]. Some CT images are shown in figure 6 below.

\section{A. CT Artifacts}

There are several artifacts that may arise in CT images. These artifacts are classified as patient-based artifacts, physics-based artifacts, and hardware-based artifacts. Patient-based artifacts include motion artifact and transient interruption of contrast. Physics-based artifacts include beam hardening, partial volume averaging, quantum mottle, photon starvation and aliasing in CT. Hardware-based artifacts include ring artifacts, tube arcing and out of field artifact [36]. CT scan has some advantages and disadvantages:

\section{ADVANTAGES}

1. CT reduces need for exploratory surgeries.

2. CT can help diagnose cancer and guide treatment.

\section{DisADVANTAGES}

1. The cancer risks are generally greater than other imaging techniques as it uses higher doses of radiation than simple $\mathrm{X}$-ray.

2. Injection of a contrast medium can cause kidney problems or allergies in some people.

3. CT scan may require anesthesia in some procedures.

\section{MAGNETIC Resonance IMAGING (MRI)}

Magnetic Resonance Imaging uses powerful magnetic fields, radio waves and properties of hydrogen atoms to produce detailed images of internal body organs, soft tissues, bones, cartilage and ligaments. Light grey or white areas in an MRI image are referred as high signal while as dark grey or black areas in an MRI image are referred to as low signal.MRI images are used to detect many types of tumors like brain tumor and abdomen tumors [37-39]. MRI images can help doctors to determine whether a tumor is noncancerous or cancerous. MRI also aids in learning about stages of cancer and planning treatments like surgery and radiation therapy and also helps in monitoring the response of treatment. Some MRI images are shown in figure 7 below.

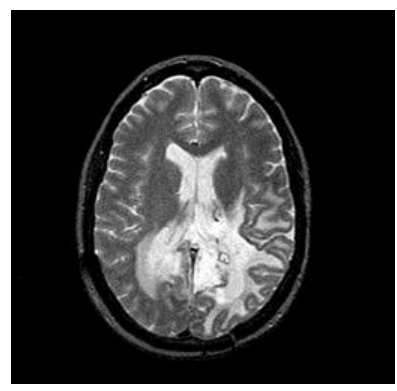

(a)

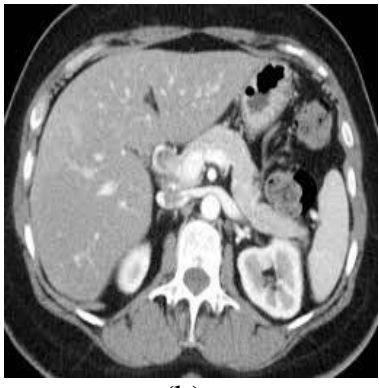

(b)

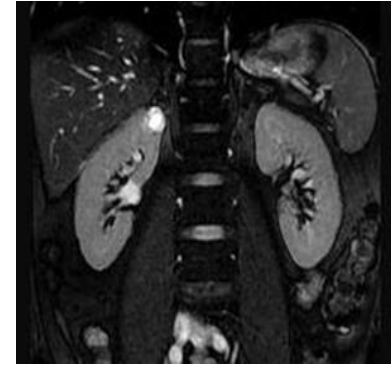

(c)

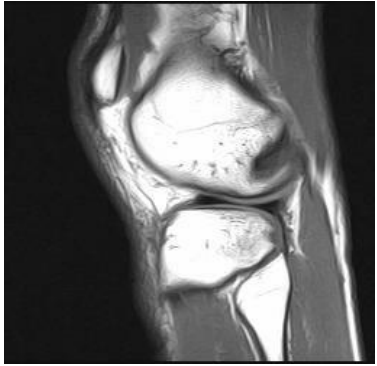

(d)
Fig.7. (a) MRI Image of Brain, (b)MRI Image of Liver, (c) MRI Image of Kidney, (d) MRI of Knee

\section{A. MRI Artifacts}

There are various artifacts associated with MRI technology which can degrade image quality and can lead to inaccurate diagnosis. MRI artifacts include Motion artifacts which arise due to movement of object or part of body during imaging. Motion artifacts include Respiratory motion, Cardiac motion, and vascular pulsation. Other MRI artifacts are Susceptibility artifacts, Chemical Shift artifacts, wrap around artifacts, partial volume artifacts, Gibbs Ringing artifacts, Cross excitation, zipper, shading artifacts. MR artifacts cause image degradation. Investigations of MRI artifacts lead to further understanding of image processing and help in enhancing the MRI images for diagnosis. MRI artifacts can be broadly grouped into two categories first, artifacts related to hardware which needs service personnel for correction. The second category is patient related artifacts. These artifacts can be corrected easily once they are recognized [40-41]. There are some advantages and disadvantages of MRI which are as under:

\section{ADVANTAGES}

1. MRI procedure is painless

2. It does not use ionizing radiations.

3. Soft tissue contrast is excellent.

\section{DISADVANTAGES}

1. MRI is a time consuming procedure.

2. It is noisy.

3. It may require anesthesia for children.

4. Movement during MRI procedure can affect the MRI images.

5. Movements during MRI can ruin the image, and may need to retest.

6. Some people may feel claustrophobic during MRI scan.

7. Contrast medium Injection can cause kidney problems.

\section{Optical Imaging (OI)}

Optical imaging uses visible light, ultraviolet, or infrared light to pass through the tissue to assess its optical properties by the detector. Various optical imaging devices are used for the detection of changes in blood volume, flow rates, endogenous tissue, fluorescent signals 
and tissue components. Tumor tissues are highly hypoxic and have altered blood flow, difference in optical absorbance of oxy- and de-oxy-hemoglobin in the tumor and normal tissues can be used to indicate the presence of lesion. Advanced computer algorithms are used to construct images. There are various techniques of optical imaging to obtain images of tissues and cells of the body like Endoscopy, Optical Coherence Tomography (OCT), diffuse optical tomography (DOT), Raman spectroscopy, super-resolution microscopy, Terahertz tomography etc. Optical imaging has the potential to improve the outcome of cancer surgery, minimize anesthesia time of patients and lower healthcare costs by improved contrast and depth of tissue penetration [42-44]. Some OI images are shown in figure 8 below.

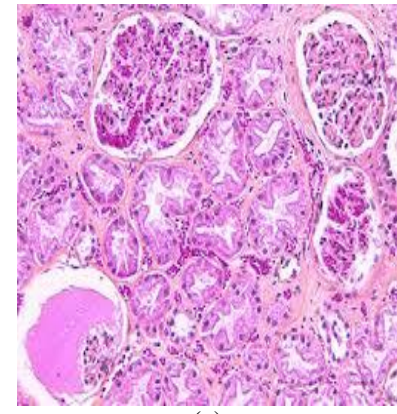

(a)

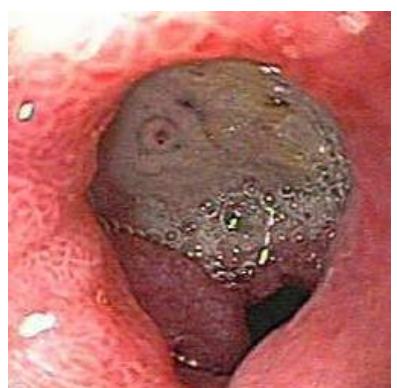

(c)

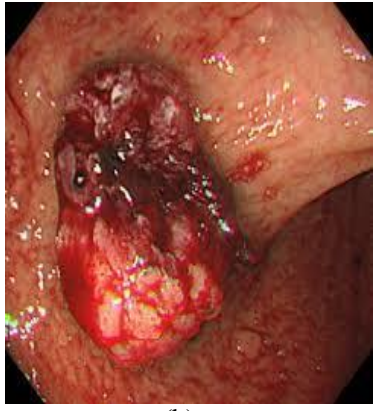

(b)

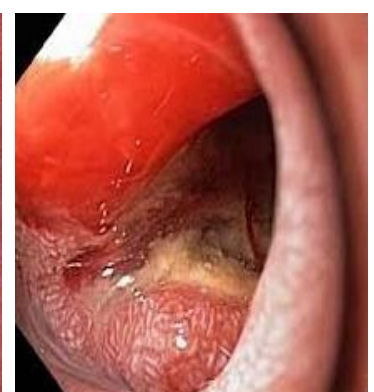

(d)
Fig.8. (a) Cancer Cells, (b) Stomach Cancer, (c) (d) OI of Duodenum

\section{A. Optical imaging Artifacts}

During optical imaging artifacts produced may cause distortion of image and can affect various areas where there is a need to focus for diagnosis, The artifacts are Motion artifacts, axial flattering artifacts, Scanning beam distortion, imaging artifacts, background noise. There are some techniques by which we can correct these artifacts for better results and for early diagnosis [45-46] [52]. There are some advantages and disadvantages of IO which are as under:

\section{ADVANTAGES}

1. Optical imaging is safe as it does not use radioactive components.

2. Optical imaging is not costly as compared to some other imaging techniques like MRI, CT scan etc.

3. Optical imaging has high sensitivity of signal detection.

4. Optical imaging can be used to visualize soft tissues.
5. Optical imaging has short imaging time as compared to other imaging techniques.

6. Optical imaging can be used for repeated studies over time.

\section{DISADVANTAGES}

1. Optical imaging suffers from signal attenuation for deeper tissues.

\section{CONCLUSION}

The paper discusses imaging techniques for cancer diagnosis. Various imaging techniques exist to develop images of the interior structure of human body for diagnosis of various diseases. These imaging techniques vary in the technology they use and their application. Xrays are used to diagnose bone cancers. Mammograms are used to detect breast cancer. PET and SPECT can be effective to diagnose early stages of cancer. CT can be used to detect tumors and for treatment guidance. Each imaging techniques has some advantages and disadvantages. US and MRI are safe than that of some other imaging techniques like X-ray and CT scan. There is risk of cancer in CT scan and X-ray due to exposure to ionizing radiations. Various artifacts exist in these imaging techniques and the images they produce for diagnosis which can lead to wrong diagnosis or difficulty in early diagnosis of cancer. These artifacts need to be recognized so that we can make corrections accordingly to produce better results as far as cancer diagnosis is concerned.

\section{ACKNOWLEDGMENT}

The authors would like to thank head of the department and other members of Radiology department Sher-iKashmir Institute of Medical Sciences, Srinagar for their support and encouragement.

\section{REFERENCES}

[1] Weinstein, R. S., Graham, A. R., Richter, L. C., Barker, G. P., Krupinski, E. A., Lopez, A. M., ... \& Gilbertson, J. R. (2009). Overview of telepath logy, virtual microscopy, and whole slide imaging: prospects for the future. Human pathology, 40(8), 1057-1069.

[2] Khalkhali, I., Mena, I., \& Diggles, L. (1994). Review of imaging techniques for the diagnosis of breast cancer: a new role of prone scintimammography using technetium$99 \mathrm{~m}$ sestamibi. European journal of nuclear medicine, 21(4), 357-362.

[3] Astrakhantsev FA, Ivanov AV, Erdneeva NV, "X-ray diagnosis of lung cancer and its morphological variants" Vestn Rentgenol Radiol. 2004 May-Jun;(3):26-31.

[4] Pietrobelli, A. N. G. E. L. O., Formica, C. A. R. M. E. L. O., Wang, Z. I. M. I. A. N., \&Heymsfield, S. B. (1996). Dual-energy X-ray absorptiometry body composition model: review of physical concepts. American Journal of Physiology-Endocrinology And Metabolism, 271(6), E941-E951.

[5] Koningsberger, D. C., \&Prins, R. (1988). X-ray absorption: principles, applications, techniques of EXAFS, SEXAFS, and XANES. 
[6] Neill Serman, Radiographic errors and artifacts. August 2000.

[7] Heywang-Köbrunner, Sylvia H., Astrid Hacker, and Stefan Sedlacek. "Advantages and disadvantages of mammography screening." Breast care 6.3 (2011): 199207.

[8] Iared, W., Shigueoka, D. C., Torloni, M. R., Velloni, F. G., Ajzen, S. A., Atallah, Á. N., \& Valente, O. (2011). Comparative evaluation of digital mammography and film mammography: systematic review and meta-analysis. Sao Paulo Medical Journal, 129(4), 250-260.

[9] R.Ramani, N.Suthanthira Vanitha,"Computer A ided Detection of Tumours in Mammograms", IJIGSP, vol.6, no.4, pp.54-59, 2014.DOI: 10.5815/ijigsp.2014.04.07.

[10] Eklund GW, Cardenosa G, Parsons W. Assessingadequacy of Mammographic image quality. Radiology 1994; 190:297-307.

[11] Ayyala RS, Chorlton M, Behrman RH, et al. Digital mammographic artifacts on full-filled systems: what are they and how do I fi them? RadioGraphics 2008; 28:1999-2008

[12] Pisano ED, Cole EB, Hemminger BM, et al. Imageprocessing algorithms for Digital mammography: a pictorial essay. RadioGraphics 2000; 20:1479-1491.

[13] Richards, C. L., Viadro, C. I., \& Earp, J. A. (1998).Bringing down the barriers to mammography: a review of current research and interventions.Breast disease, 10(3-4), 33-44.

[14] Gregg, E. W., Kriska, A. M., Salamone, L. M., Roberts, M. M., Aderson, S. J., Ferrell, R. E., Cauley, J. A. (1997). The epidemiology of quantitative ultrasound: a review of the relationships with bone mass, osteoporosis and fracture risk. Osteoporosis international, 7(2), 89-99

[15] Bailey, M. R., Khokhlova, V. A., Sapozhnikov, O. A., Kargl, S. G., \& Crum, L. A. (2003). Physical mechanisms of the therapeutic effect of ultrasound (a review). Acoustical Physics, 49(4), 369-388.

[16] Matalon TA, Silver B. US guidance of interventional procedures. Radiology 1990; 174:43-47.

[17] Scanlan KA. Sonographic artifacts and their origins., AJR Am J Roentgenol; 156(6):1267-1272.

[18] Cabeza, R., \& Nyberg, L. (2000).Imaging cognition II: An empirical review of 275 PET and fMRI studies.Journal of cognitive neuroscience, 12(1), 1-47.

[19] Glaser, M., Luthra, S. K., \& Brady, F. (2003). Applications of positron-emitting halogens in PET oncology (Review). International journal of oncology, 22(2), 253-267.

[20] Maes F, Collignon A, Vandermeulen D, Marchal G,Suetens P. Multimodality image registration by maximization of mutual information. IEEE Trans MedImaging 1997; 16:187 - 98.

[21] Slomka PJ. Software approach to merging molecular with anatomic information. J Nucl Med 2004; 45:36-45S

[22] Mattes D, Haynor DR, Vesselle H, Lewellen TK, Eubank W. PET-CT image registration in thechestusing free-form deformations.IEEE Trans Med Imaging 2003;22:120 - 34 .

[23] Wes t J, Fitzpatrick JM, Wang MY, Dawan t BM, Maurer Jr CR, Kessler RM, et al. Comparison andevaluation of retrospective inter modality brain image registration techniques. J Computer AssistTomogr 1997; 21:554 - 66.

[24] West J, Fitzpatrick JM, Wang MY, Da want BM, Maurer Jr CR, Kessler R M, et al. Retrospectiveinter modality registration techniques for images of the head: surfacebased versus volume-based. IEEETrans Med Imaging 1999; 18:144 - 50 .

[25] Stearns C, Miesbauer D, Culp R. Measuring gantry and gantry-table alignment in PET-CT. In: IEEE Nuclear Science Symposium and Medical Imaging Conference Record, 2003.

[26] Beyer T, Antoch G, Blodgett T, Freudenberg LF, Akhurst T, Mueller S. Dual-modality PET-CT imaging: the effect of AC F -artifact, respiratory motion on combined image quality in clinical oncology. Eur J Nucl Med 2003;30:588 $-96$

[27] Stacy, M. R., Maxfield, M. W., \&Sinusas, A. J. (2012).Targeted molecular imaging of angiogenesis in PET and SPECT: a review.The Yale journal of biology and medicine, 85(1), 75.

[28] Rosenthal, M. S., Cullom, J., Hawkins, W., Moore, S. C., Tsui, B. M. W., \& Yester, M. (1995). Quantitative SPECT imaging: a review and recommendations by the Focus Committee of the Society of Nuclear Medicine Computer and Instrumentation Council. Journal of Nuclear Medicine, 36(8), 1489-1513.

[29] Rahmim, A., \& Zaidi, H. (2008). PET versus SPECT: strengths, limitations and challenges. Nuclear medicine communications, 29(3), 193-207.

[30] Andreas K. Buck, Stephan Nekolla, Sibylle Ziegler, Ambros Beer, Bernd J. Krause1, Ken Herrmann, Klemens Scheidhauer, Hans-Juergen Wester, Ernst J. Rummeny, Markus Schwaiger, and Alexander Drzezga:- SPECT/CT.

[31] Vercruyssen, M., Jacobs, R., Van Assche, N., \& van Steenberghe, D. (2008). The use of CT scan based planning for oral rehabilitation by means of implants and its transfer to the surgical field: a critical review on accuracy. Journal of oral rehabilitation, 35(6), 454-474.

[32] Hu, H. (1999). Multi-slice helical CT: scan and reconstruction. Medical physics, 26(1), 5-18.

[33] Atiyeh Hashemi,Abdol Hamid Pilevar,Reza Rafeh,"Mass Detection in Lung CT Images Using Region Growing Segmentation and Decision Making Based on Fuzzy Inference System and Artificial Neural Network", IJIGSP , vol.5, no.6, pp.16-24, 2013.DOI: 10.5815/ijigsp.2013.06.03

[34] Xing Li,Ruiping Wang,"A new efficient 2D combined with 3D CAD system for solitary pulmonary nodule detection in CT images",IJIGSP, vol.3, no.4, pp.18-24, 2011.

[35] Hsieh J: Computed tomography: Principles, design, artifacts, and recent Advances. SPIE,Bellingham, WA. (2003).

[36] Fleischmann D, Boas FE, Tye GA, Sheehan D, Molvin LZ: Effect of low Radiationdose on image noise and subjective image quality for analytic v/s Iterative image reconstruction in abdominal CT. In: RSNA. Chicago 2011.

[37] Dempsey, M. F., Condon, B., \& Hadley, D. M. (2002, October). MRI safety review. In Seminars in Ultrasound, CT and MRI (Vol. 23, No. 5, pp. 392-401). WB Saunders.

[38] Henkelman, R. M., Stanisz, G. J., \& Graham, S. J. (2001). Magnetization transfer in MRI: a review. NMR in Biomedicine, 14(2), 57-64.

[39] Tanuj Kumar Jhamb, Vinith Rejathalal, V.K. Govindan,"A Review on Image Reconstruction through MRI k-Space Data", IJIGSP, vol.7, no.7, pp.42-59, 2015.DOI: 10.5815/ijigsp.2015.07.06

[40] Saloner D, "Flow and motion," Magn Reson Imaging Clin N Am 1999 Nov; 7(4):699-715.

[41] Barish MA and Jara H, "Motion artifact control in body MR imaging," Magnetic Resonance Imaging Clin NAm 1999 May; 7(2):289-301.

[42] Hedley M, Yan H, "Motion artifact suppression: a review of post-processing Techniques," Magn Reson Imaging 1992; 10(4):627-35. 
[43] Elster AD, Burdette JH. Questions \& Answers in Magnetic Resonance Imaging 2nd ed. St. Louis: Mosby, 2001.

[44] Ntziachristos, V., \& Chance, B. (2000). Breast imaging technology: Probing physiology and molecular function using optical imaging-applications to breast cancer. Breast Cancer Research, 3(1), 41.

[45] Pittman, T. B., Shih, Y. H., Strekalov, D. V., \&Sergienko, A. V. (1995).Optical imaging by means of two-photon quantum entanglement.Physical Review A, 52(5), R3429.

[46] D. Huang, E. A. Swanson, C.P. lin, J.S. Schuman, W.g. Stinson, W. Chang, M.R. Flotte, K. Gregory, C.A.Puliafito, "Optical Coherence Tomography",Science 254, 1178-1181 (1991).

[47] Momose, Atsushi. "Recent advances in X-ray phase imaging." Japanese Journal of Applied Physics 44.9R (2005): 6355 .

[48] Harvey, Christopher J., et al. "Advances in ultrasound." Clinical radiology57.3 (2002): 157-177.

[49] Todd-Pokropek, Andrew. "Advances in PET and Spect." Physics for Medical Imaging Applications. Springer Netherlands, 2007. 321-340.

[50] Pathak, Arvind P., et al. "Molecular and functional imaging of cancer: advances in MRI and MRS." Essential Bioimaging Methods (2009): 183.

[51] Choy, Garry, Peter Choyke, and Steven K. Libutti. "Current advances in molecular imaging: noninvasive in vivo bioluminescent and fluorescent optical imaging in cancer research." Molecular Imaging 2.4 (2003): 303-312.

[52] M. Wojtkowski, T. Bajraszewski, P. Targowski and A. Kowalczyk "Real time in vivo imaging by highspeedspectral optical coherence tomography", Opt. Lett. 28, 1745-1747 (2003)

[53] Ishikawa, Tetsuya, et al. "A compact X-ray free-electron laser emitting in the sub-angstrom region." Nature Photonics 6.8 (2012): 540-544.

[54] Glantz, Chris, and Leslie Purnell. "Clinical utility of sonography in the diagnosis and treatment of placental abruption." Journal of ultrasound in medicine 21.8 (2002): 837-840.

[55] Penninck, Dominique. Atlas of small animal ultrasonography. John Wiley \& Sons, 2015.

[56] Van der Vaart, M. G., et al. "Application of PET/SPECT imaging in vascular disease." European Journal of Vascular and Endovascular Surgery 35.5 (2008): 507-513.

[57] Dueholm, Margit, and Erik Lundorf. "Transvaginal ultrasound or MRI for diagnosis of adenomyosis." Current Opinion in Obstetrics and Gynecology19.6 (2007): 505512.

[58] Gobin, André M., et al. "Near-infrared resonant nanoshells for combined optical imaging and photothermal cancer therapy." Nano letters 7.7 (2007): 1929-1934.

\section{Author's Profiles}

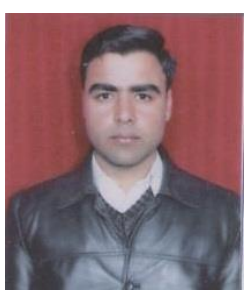

Showkat Hassan Malik is Ph.D research scholar at the Mewar University Rajasthan India in the field of image processing. He received his BCA and MCA from university of Kashmir Srinagar India. He has few publications in conferences and journals. He has worked as lecturer at PG department of computer Science University of Kashmir

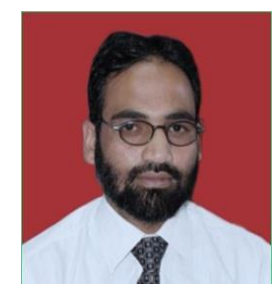

Tariq Ahmad Lone received his MCA from university of Jammu India. He is $\mathrm{Ph} . \mathrm{D}$ candidate at Mewar University Rajasthan India. $\mathrm{He}$ is working as Assistant professor at Business School University of Kashmir India since 2007. $\mathrm{He}$ has contributed to conferences and journals in the field of computer science. His research interests include image processing and database systems.

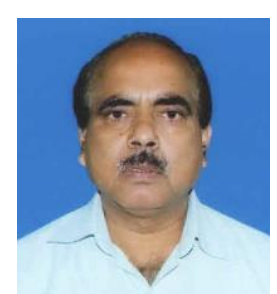

Prof. S. M. K Quadri is working as Professor at the PG department of computer Science Jamia Millia Islamia New Delhi India. He received his Ph.D from university of Kashmir in 2008. His research interests include Software Engineering, Software Reliability Engineering and image processing. He has authored more than 100 manuscripts in the field of computer science. He has guided more than $20 \mathrm{Ph} . \mathrm{D}$ students and Has 24 years of teaching experience at University of Kashmir. 